\title{
Wolfgang Rother Zur Ontologie von Lust und Leiden
}

I.

Lust und Leiden sind Empfindungen und als solche subjektiv.

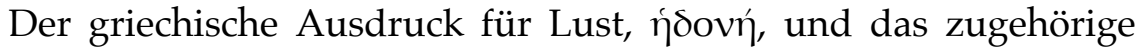

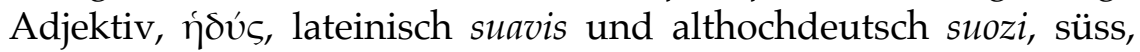
verweisen in den Bereich des Geschmacks und des Geruchs - bei Homer, um ein frühes Zeugnis zu erwähnen, beispielsweise des angenehm süss duftenden Weines. ${ }^{1}$ Leiden, lateinisch patior mit der Substantivierung passio oder griechisch $\pi \alpha \dot{\alpha} \sigma \chi \omega$, ist das Erleben, Erfahren und Erleiden dessen, was mir zustösst oder widerfährt bei Homer steht in einem Vers über die griechischen Krieger die Differenz zum Tun und Handeln im Blick, ${ }^{2}$ nicht zur Lust, die sich diesbezüglich nicht notwendig vom Leiden unterscheidet, denn auch Angenehmes, auch eine Lustempfindung kann durchaus passiv erfahren werden, doch ist Leiden im Unterschied zur Lust negativ konnotiert und bezeichnet insbesondere das Erdulden eines Übels.

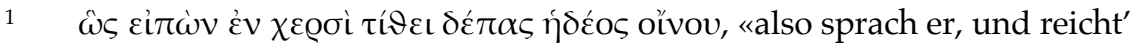
ihr den Becher voll duftenden [wörtlich: süssen] Weines» Hom. Od.

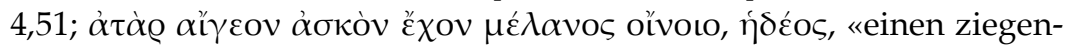
ledernen Schlauch auf der Achsel, voll schwarzen süssen Weines» Hom. Od. 9,196-197 (übers. von Johann Heinrich Voss).

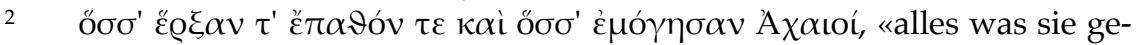
tan und erduldet im mühsamen Kriegszug» Hom. Od. 8,490 (übers. von J. H. Voss). 
Lust ist also ursprünglich die durch den Geschmacks- oder Geruchssinn, aber auch durch Tastsinn, Gehör und Sehen vermittelte angenehme Empfindung, die wir, wenn sie nicht das physische, sondern das psychische Erleben betrifft, als Freude bezeichnen. Leiden hingegen ist die Empfindung, die sich einstellt, wenn wir etwas Unangenehmes erleben, wenn uns körperlicher oder seelischer Schmerz zugefügt wird.

Als subjektive Empfindungen sind Lust und Leiden unmittelbar erleb- und erfahrbar, aber nicht adäquat mitteilbar. Wenn ich sage, dass ich Lust empfinde oder dass ich leide, werde ich zwar verstanden, weil alle Menschen Lust und Leiden empfinden können beziehungsweise ebenfalls sagen, dass sie solche Empfindungen haben, aber die anderen Menschen wissen nicht, wie sich meine Lust und mein Schmerz anfühlen, genauso wenig, wie ich je werde erfahren können, was ein anderer Mensch empfindet, wenn er sagt, dass er sich über etwas freue oder physisch beziehungsweise psychisch leide. Diese genuine Subjektivität von Lust und Leiden scheint gegen ihre ontologische Dignität zu sprechen. Wenn ich daher dennoch von einer «Ontologie von Lust und Leiden» spreche, will ich damit sagen, dass nicht nur der Gegenstand von Erkenntnis und Empfindung ein Seiendes ist, sondern auch Bewusstsein, Erkenntnis und Empfindung, die als Seiendes in einem psychophysischen Prozess «in die Gesamtsphäre des Seienden eingebettet» sind und insofern wahr sind, als sie einen «Wahrheitskern» haben und «Zeugnis» ablegen «von einem wirklichen seienden Objekt». ${ }^{3}$

Was in den folgenden Bemerkungen zur Ontologie von Lust und Leiden versucht wird, ist eine grobe Skizzierung der komplexen Grundstrukturen und Einteilungen dieser Empfindungen. Nach kurzen Hinweisen zur Etymologie und Sprachgeschichte werden Lust und Leiden aus unterschiedlichen Perspektiven beleuchtet. Zunächst wird in einer semantisch-sprachanalytischen

3 Vgl. Nicolai Hartmann: Grundzüge einer Metaphysik der Erkenntnis (1921), Berlin 51965, 393-403 (52. Kapitel. Der ontologische Sinn der Empfindung), hier 394 u. 396. 
Annäherung untersucht, welche Empfindungen wir haben, wenn wir in verschiedenen Kontexten und Situationen von Lust und Leiden sprechen. Dabei werden unterschiedliche Gestalten und Facetten dieser Empfindungen thematisiert. Schliesslich werden der ontologische Status sowie das ontologische Ineinanderverschlungen-sein von Lust und Leiden in verschiedenen Konstellationen diskutiert.

II.

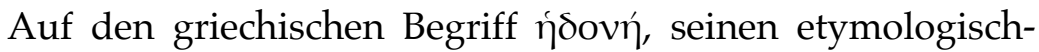
semantischen Kontext und seine Herkunft aus dem GustatorischKulinarischen und Olfaktorischen wie auch die Ausweitung auf alle Bereiche angenehmer sinnlicher Empfindungen haben wir bereits hinwiesen. In hellenistischer Zeit, namentlich im Neuen Testa-

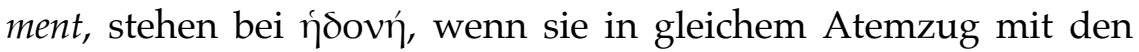
sinnlichen Begierden, غ̇ंı $\theta v \mu$ í $\alpha \mathrm{l}$, genannt wird, zweifellos auch die negativ konnotierte Wollust und Geschlechtslust im Blick. ${ }^{4}$ Dass das deutsche Wort Lust im neutestamentlichen Kontext weitgehend auf sexuelle Lust reduziert wird, dürfte auf Luthers Übersetzung von غ̇ंı $\theta v \mu i ́ \alpha$ mit Lust statt mit Begierde zurückzuführen sein, ${ }^{5}$ was sich bis ins evangelische Liedgut des 17 . Jahrhunderts

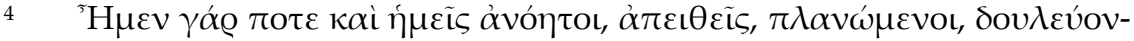

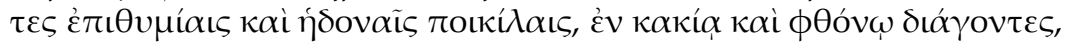

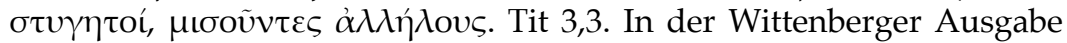

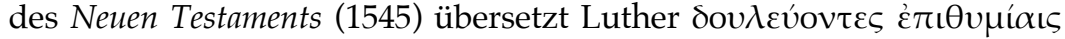

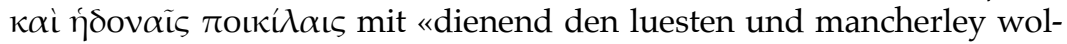
luesten».

5 Luther übersetzt غ̇ंı $\theta v \mu$ í $\alpha$ mit Lust, beispielsweise Röm 7,7 u. 7,8; Gal

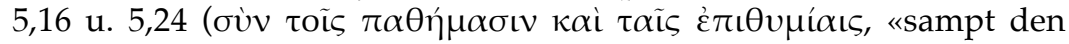

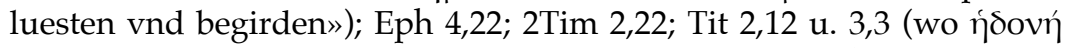
mit Wollust übersetzt wird), 2Petr 1,4; 2,10 u. 2,18; 1Joh 2,16 u. 2,17. Entsprechend auch Jacob und Wilhelm Grimm: Deutsches Wörterbuch, Leipzig 1854-1960, XII 1314-1316: Lust als «heftiges verlangen, begierde» mit dem Hinweis, dass «die bibel die lust in beziehung zur sünde bringt» und der ebenfalls dieser Perspektive zu verdankenden 
fortpflanzt, wo die Lust der Inbegriff eines weltlichen, gottabgewandten und sündigen Lebens ist. ${ }^{6}$

Sprachgeschichtlich geht «Lust», gotisch lustus, wahrscheinlich auf griechisch $\lambda \alpha \alpha(\sigma) \omega$, packen, ergreifen, wollen, und lateinisch lascivus zurück. ${ }^{7}$ Das Wort bedeutet soviel wie lose, locker, zunächst im Sinne eines harmlos-neckischen Schäkerns, dann aber auch im Sinne von übermütig, zügellos und geil, also bereits dessen, was wir heute unter «lasziv» verstehen. ${ }^{8}$ So lässt sich aus der sprachgeschichtlichen Betrachtung Lust als ein Wollen und Begehren deuten, das unterschiedliche Stufen und Facetten vom scherzendspielerischen Getändel bis hin zum Exzess der Unbeherrschtheit, Zügellosigkeit und Laszivität umfasst. Diese auf das griechische $\lambda \alpha ́(\sigma) \omega$ zurückgehende Verwandtschaft von Lust und Wollen oder Wille wurde auf die romanischen Sprachen vererbt. Das Verb volo, wollen, willens sein, begehren, wünschen, kennt zwei unterschiedliche Substantive: die voluntas als den Willen, der eine geistige Richtung bezeichnet und speziell den eigenen, freien und guten Willen, und die voluptas, die nicht nur das Vergnügen, den Genuss und die Lust im guten wie üblen Sinne bezeichnet, sondern auch den Hang zum sinnlichen Vergnügen, in dem der Wille nicht mehr frei, sondern gewissermassen triebgesteuert und insofern fremdbestimmt ist. ${ }^{9}$ Vergleichbare Differenzen und Verbindungen kennt auch das Italienische: Das von volere, wollen, abgeleitete Substantiv voglia ist die Lust auf etwas, das Begehren und namentlich die sexuelle Begierde, wie denn auch das Adjektiv voglioso, lüstern, in

Information, dass die Pluralform Lüste «seit alters gern zur bezeichnung heftiger und mannigfaltiger sinnenreize» stehe.

6 «Die Lust, die unser Fleisch ergötzt, / Die zeucht uns nach der Höllen, / Und was die Welt für Freude schätzt, / Pflegt Seel und Geist zu fällen / Und ewiglich zu quälen.» Paul Gerhard: O Gott, mein Schöpfer, edler Fürst (1647), in: Dichtungen und Schriften, hg. und textkritisch durchgesehen von Eberhard von Cranach-Sichart, München 1957, 159.

7 Grimm, XII 1314.

8 Karl Ernst Georges: Ausführliches lateinisch-deutsches Handwörterbuch, Hannover 1913-1918, s.v. lascivus.

9 Georges, s.v. volo [2], voluntas, voluptas. 
den gleichen Kontext des Libidinösen gehört, während die volontà wie im Lateinischen den freien Willen bezeichnet. Während voglia die Lust auf etwas bezeichnet (avere voglia di qualcosa), wird die Lust oder das Gefallen an etwas piacere, von lateinisch placeo, genannt, eine Differenz, die auch das Englische kennt, wo die Verwendung von lust auf die sinnliche und sexuelle Begierde eingeschränkt ist, während das romanische Lehnwort pleasure Lust im Sinne von Vergnügen, Freude und Gefallen bedeutet. ${ }^{10}$

Die Sprachgeschichte des Wortes «Leiden» oder «leiden» wartet mit einer kleinen Überraschung auf. Im Gotischen, Altsächsischen, Angelsächsischen und Altnordischen bedeutet das Verb nämlich «gehen», «reisen» und besonders «mit dem Schiff fahren», während es erst im Althochdeutschen, das heisst in der zweiten Hälfte des 9. Jahrhunderts, auch im heutigen Sinne von «dulden» verwendet wird. ${ }^{11}$ Eine Spur in den lebensweltlichen Kontext dieser Bedeutungsverschiebung legt die Etymologie von «Elend», das ursprünglich das Wohnen im Ausland und das damit verbundene Leiden in der Fremde, das Heimweh, bezeichnete. ${ }^{12}$

Paradigmatisch für den intransitiven Gebrauch des Verbs ist das Leiden Christi, das passive Erdulden von Not und Schmerz im Gegensatz zum aktiven Handeln. ${ }^{13}$ Hier steht wiederum die Differenz von patior, ein Leiden über sich ergehen lassen, und passio auf der einen Seite und ago, etwas durch das Handeln in Bewegung setzen, und actio auf der anderen Seite im Blick. Das schliesst indes nicht aus, dass auch das Tätigsein mit Leiden verbunden ist. Und umgekehrt kennt die deutsche Sprache nicht nur «leiden» in passiver Bedeutung, sondern auch im aktiven Sinn von lieb- und gernhaben, wenn ich beispielsweise sage, dass ich jemand oder etwas gut leiden mag oder eben nicht leiden kann. ${ }^{14}$

Vgl. W. Rother: Lust. Perspektiven von Platon bis Freud, Basel 2010, 8-9.

Grimm, XII 658.

Grimm, III 406.

Grimm, XII 659 u. 666-667.

Vgl. Grimm, XII, 662-664. 
Eine weitere überraschende Wende nimmt das archetypische Leiden, das heisst die Passion Christi als Inbegriff höchster Tugend, mit der wahrscheinlich im 17. Jahrhundert erfolgten Bildung des Kollektivbegriffs «Leidenschaft», mit dem die französische passion und die italienische passione dell'animo übersetzt wurden und der seit dem 18. Jahrhundert eine «heftige Seelenregung» bezeichnet, «deren Grund eine drängende sinnliche Begierde ist», ${ }^{15}$ die für Kant ganz und gar keine Tugend, sondern «ein wahres Laster» darstellt. ${ }^{16}$ Der Bedeutungswandel der Passion von der höchsten Tugend zum genuinen Laster und vom Leiden zur Leidenschaft zeigt, wie fliessend die Grenzen sind zwischen den Sphären des Leidens und der Lust, deren Ineinander-verschlungen-sein Goethe nach der Erfahrung einer Liebe, die unerwidert blieb, in seiner Trilogie der Leidenschaft zum Ausdruck bringt: «Die Leidenschaft bringt Leiden!» ${ }^{17}$

III.

Im sprachgeschichtlichen Stamm des Wortes Lust, $\lambda \alpha \dot{\alpha}(\sigma) \omega$ im Sinne von packen, ergreifen, wollen, und in der Semantik von

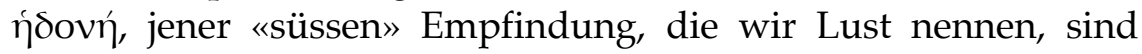
zwei Grundbedeutungen von Lust angelegt, die Lust auf etwas und die Lust an etwas, die ich andernorts als intentionale und aktual-performative Lust bezeichnet habe. ${ }^{18}$ Die Lust-auf gründet in einem Bedürfnis, also in einem Mangel, der in der Regel als Schmerz empfunden wird, und äussert sich in einem Begehren,

15 Grimm, XII 670.

16 Kant: Metaphysik der Sitten, Akademie-Ausgabe, VI 408.

17 Goethe: Werke, Hamburger Ausgabe, I 385. Vgl. auch den Aufsatz von Ulrike Zeuch: Goethes Trilogie der Leidenschaft, in der vorliegenden Nummer von conexus, 45-64.

18 W. Rother: Lust, 9; ders.: Genießen und Genuss. Annäherungen an ein Phänomen menschlicher Existenz, in: Lothar Kolmer, Michael Brauer (Hg.): Hedonismus. Genuss - Laster - Widerstand?, Wien 2013, 15-28, hier 20 . 
das auf die Befriedigung jenes Bedürfnisses gerichtet ist. Was ich im Prozess der Befriedigung des Bedürfnisses empfinde, ist aktualperformative Lust. Dieser Lustprozess endet im Moment der Befriedigung des Bedürfnisses: Ist der Hunger gestillt, der Durst gelöscht, der sexuelle Höhepunkt erreicht, ist also die intentionale Lust am Ziel angekommen, empfinden wir nicht mehr, was wir im Deutschen als Lust, sondern das, was wir als Zufriedenheit oder Wohlbefinden bezeichnen.

Dieses Ziel benennt Epikur hingegen explizit als Lust, nämlich

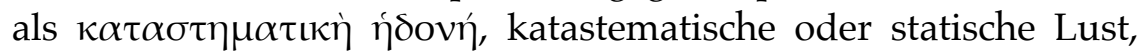

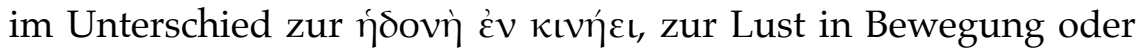
kinetischen Lust. ${ }^{19}$ Die kinetische Lust umfasst die Phasen der intentionalen und der aktual-performativen Lust, das Begehren und den Genuss bei der Befriedigung des Bedürfnisses, die beide eine Bewegung darstellen, welche in der katastematischen Lust endet, jenem Zustand der Ruhe, in dem wir uns befinden, wenn die als Schmerz empfundene Lust-auf im Prozess der Lust-an befriedigt worden ist. Die katastematische Lust ist also zugleich das Ziel und das Ende der Lust. Wird dieses Ziel überschritten, schlägt Lust in Unlust oder Schmerz um. So wie am Anfang der Schmerz als Empfindung des unbefriedigten Bedürfnisses stand, stellen sich ebenfalls Schmerz, Unlust und Überdruss ein, wenn ich zu viel des Guten genossen habe. ${ }^{20}$

Angesichts der von Epikur geltend gemachten Differenz zwischen kinetischer und katastematischer Lust stellt sich die Frage, ob es auch so etwas wie kinetisches und katastematisches Leiden gebe. In gewisser Weise finden sich Analogien zu dieser Differenz in den von mir andernorts diskutierten Unterschieden zwischen aktuellem und habituellem Leiden sowie zwischen dem durch etwas Bestimmtes und dem grundlosen, durch nichts (oder das Nichts) ausgelösten Leiden an existentieller Lebensunlust, die

19 Diog. Laert. 10,136.

20 Vgl. dazu W. Rother: Facetten der Unlust, in: Figurationen. Gender Literatur - Kultur 15/ 2 (2014) 16-30, hier 18 u. 20-21. 
Menschen in den Suizid treiben kann. ${ }^{21}$ Aktuelles und temporäres, zeitlich begrenztes Leiden wie auch das Leiden, das eine konkrete Ursache hat, sind in gewisser Hinsicht kinetisches Leiden. Dieses kann sich im Prozess des Leidens steigern, aber aktuell-temporäres Leiden geht vorüber und findet ein Ende. Wenn die Ursache des Leidens etwas Bestimmtes ist, kann die Ursache bekämpft werden. Leiden hingegen, das habituell ist, das Leiden an der Existenz, das keine konkrete Ursache hat, jene existentielle Leere, jener «ennui», dem Baudelaire in den Fleurs du mal, oder jener Lebensekel, dem Sartre in La Nausée literarischen Ausdruck verliehen hat, sind katastematische Grundbefindlichkeiten.

Zurück zur katastematischen Lust, die wir als das Ende der Lust bezeichnet haben. Es gibt gleichwohl noch eine Lust nach diesem Ende der Lust, nämlich die Lust, die aus der Sphäre der Empfindung in die des Bewusstseins gehoben wird. Im Prozess der aktual-performativen Lust empfinden wir nicht nur Lust, sondern wir sind uns bewusst, dass wir Lust empfinden. Dank diesem Bewusstsein können wir sagen, dass wir (etwas) geniessen. Insofern geniessen wir nicht mit unseren Sinnesorganen, sondern mit unserem Geist. Es gibt daher auch kein bewusstloses Geniessen. Vielmehr ist das Geniessen ein Phänomen des Bewusstseins, das die sinnliche Lustempfindung nicht nur vergegenwärtigt und damit steigert, sondern auch aufbewahrt und erinnerbar macht, so dass die Erinnerung an empfundene sinnliche Lust ebenso mit Lustempfindungen verbunden ist und als wirklicher Genuss erlebt wird wie die erinnerte Lust selbst. Ich habe andernorts mit Bezug auf das, was Sisyphos in der Interpretation von Camus als «Stunde des Bewusstseins» erfährt, ${ }^{22}$ dafür argumentiert, dass Genuss nicht nur vom Bewusstsein derjenigen, die geniessen, begleitet wird, sondern in diesem Bewusstsein, das heisst dem bewussten Erleben von Lustempfindungen besteht. ${ }^{23}$

21 Ebd., 17-18 u. 21-23.

22 Albert Camus: Der Mythos des Sisyphos, übers. von Vincent von Wroblewsky, Reinbek bei Hamburg 1999, 157.

23 Vgl. W. Rother: Genießen und Genuss, 21-22. 
Dass es die Vorstellung selbst ist, die wir als lustvoll erleben, und nicht die in der Vergangenheit erlebte, also die erinnerte Lustempfindung, hat Gustav Theodor Fechner mit Blick auf Inhalte lustvoller Vorstellungen gezeigt, die weder in der Vergangenheit noch in der Gegenwart, sondern in der Zukunft liegen und insofern gar keine wirklich erlebten Lustempfindungen sein können. ${ }^{24}$ Dass nicht die zukünftige Lust im Moment, in dem ich sie mir vorstelle, lustvoll ist, sondern die Vorstellung selbst, und dass diese lustvolle Vorstellung stärker als real empfundenes Leiden sein kann, hat Fechner an Fällen aufgezeigt, in der die zukünftige Lust gar nicht mehr empfunden werden konnte, da das Leiden zum Tod führte.

Lustvoll für den Soldaten Marcus Curtius, der der bei Titus Livius überlieferten Sage zufolge sein Leben opferte, um seine Vaterstadt Rom zu retten, ${ }^{25}$ war nicht die erhoffte Rettung Roms oder der erwartete mögliche Nachruhm, der ebenso unsicher war wie die Rettung Roms und dessen er sich, wenn er tatsächlich eingetreten wäre, gar nicht hätte erfreuen können, sondern es waren einzig die Vorstellung von der Rettung Roms und der Gedanke an den eigenen Ruhm, die für ihn lustvoll waren. Ebenso empfand Christus die Vorstellung, dass er durch sein Leiden und Sterben die Menschheit erlösen wird, als lustvoll, nicht das dadurch bewirkte Heil der Welt selbst. ${ }^{26}$ Insofern ist es nicht die «Vorstellung

24 Vgl. Gustav Theodor Fechner: Ueber das Lustprincip des Handelns, in: Zeitschrift für Philosophie und philosophische Kritik, Neue Folge 19 (1848) 1-39, 163-194, hier 5 u. 17-18. Dazu W. Rother: «Vorstellung der Lust oder Lust der Vorstellung», in: ders.: Lust, 140-142; ders.: Hedonismus und Ästhetik. Bemerkungen zu Gustav Theodor Fechner, in: Leitmotiv. Topics in Aesthetics and in Philosophy of Art. Motivi di estetica e di filosofia dell'arte, n.s. 0 (2010) 71-87, hier 83-84

www.ledonline.it/leitmotiv/Allegati/Leitmotiv-2010-0-Rother.pdf;

ders.: An den Grenzen hedonistischer Lebenskunst. Leiden, Schmerz, Trauer, Krankheit und Tod, in: Lothar Kolmer, Michael Brauer (Hg.): Hedonismus leben. Der "gelungene Tag» in Geschichte und Gegenwart, Wien 2016, 29-40, hier 36-37.

25 Liv. ab urbe condita 7,6.

26 Vgl. G. Th. Fechner: Ueber das Lustprincip des Handelns, 6-7. 
der Lust», die unser Handeln motiviert, sondern die «Lust der Vorstellung». ${ }^{27}$

In Bezug auf Schmerz und Leiden sind die Erinnerung an erfahrenes Leid oder die Vorstellung zukünftigen Schmerzes in ähnlicher Weise wirksam, wobei die Vorstellung des Leidens und das Leiden an einer Vorstellung ineinander verschränkt sind. Die Erinnerung an das traumatisierende Erlebnis wird als wirklicher Schmerz erfahren, wie auch die Vorstellung eines Schmerzes oder Leidens, das die Zukunft betrifft und mich insofern gar nicht unmittelbar affiziert, ein Gefühl der Angst hervorruft, an dem ich wirklich leiden kann.

Die oben geltend gemachte Differenz zwischen intentionaler und aktual-performativer Lust lässt sich in analoger Weise bei der Unlust beobachten. ${ }^{28}$ Intentionale Unlust ist das zielbewusste Nichtbegehren von etwas und äussert sich in einem aversiven Reflex, der - wie die Lust auf etwas - unterschiedlich stark ausgeprägt sein kann. Er reicht vom Keine-Lust-auf-etwas-haben bis hin zum Ekel vor etwas und Angewidert-sein von etwas. Das negative Pendant zur aktual-performativen Lust ist die Frustration, die wir empfinden, wenn die intentionale Lust nicht zum Ziel gelangt, wenn uns das Begehrte verweigert wird oder wenn der Prozess der Lustbewegung abgebrochen wird.

Das Leiden hingegen kennt die Differenz zwischen intentional und aktual-performativ nicht. Leiden als Erdulden physischer und psychischer Schmerzen ist ausschliesslich aktual-performativ. Das Ende des Leidens kann gewollt werden, aber nur durch Aufgabe jener das Leiden kennzeichnenden Passivität, indem der Leidende das Ende seines Leidens will, es nicht wie der leidende Christus in seinem Willen zum Leiden erträgt und erduldet, sondern indem er dagegen protestiert und rebelliert. Für Nietzsche ist das Leiden das eigentliche Lebenselixier, die Antriebkraft zum Leben. ${ }^{29}$ Das

Ebd., 17-18 u. 29.

Vgl. W. Rother: Facetten der Unlust, 19.

«Die normale Unbefriedigung unsrer Triebe z.B. des Hungers, des Geschlechtstriebs, des Bewegungstriebs, enthält in sich durchaus noch 
Leiden «stärkt den Willen zur Macht». ${ }^{30}$ Was Nietzsche hier als Wille zur Macht bezeichnet, ist nichts anderes als die intentionale Lust, jener Wille zur Lust, der Leiden und Schmerz überwinden will. Lust ist für Nietzsche mit dem «Gefühl der Macht» und dem «Bewußtsein der Macht» verbunden und setzt Unlust und Leiden voraus. ${ }^{31}$ Insofern sind Lust und Leiden Antagonisten - der Wille zur Lust ist der Wille, dass das Leiden aufhöre.

IV.

Und wenn das Leiden aufhört, empfinden wir Lust. Genau diese These vertritt Pietro Verri. Lust ist in seiner Perspektive nichts anderes als das, was wir empfinden, wenn Schmerz und Leiden plötzlich aufhören. ${ }^{32}$ Er stellt damit die Ontologie von Lust und Leiden auf den Kopf. Jedes Gute hat für ihn seine Wurzel in einem Übel. ${ }^{33}$ Dies bedeutet eine Dementierung der plotinisch-

nichts Herabstimmendes; sie wirkt vielmehr agacirend auf das Lebensgefühl, wie jeder Rhythmus von kleinen schmerzhaften Reizen es stärkt [...]: diese Unbefriedigung, statt das Leben zu verleiden, ist das große Stimulans des Lebens.» Friedrich Nietzsche: Nachgelassene Fragmente (1887-1889) 11 [76], in: Kritische Studienausgabe, XIII 38.

$30 \quad$ Ebd. 11 [77], XIII 38.

31 «Die Lust tritt auf, wo Gefühl der Macht / Das Glück in dem herrschend gewordenen Bewußtsein der Macht und des Siegs», ebd. 14 [70], XIII 254; «Lust als Machtgefühl (die Unlust voraussetzend)», ebd., (1885-1887) 5 [64], XII 209.

32 Pietro Verri: Discorso sull'indole del piacere e del dolore (1781), in: I «Discorsi» e altri scritti degli anni Settanta. Edizione Nazionale, III Roma 2004, 63-152, vgl. hier 84-86, 104 u. 146-147.

33 «ogni bene nel mondo ha la sua radice nel male» P. Verri: Discorso sull'indole del piacere e del dolore, 137. Vgl. hierzu generell W. Rother: La maggiore felicità possibile. Untersuchungen zur Philosophie der Aufklärung in Nord- und Mittelitalien, Basel 2005, 88-97, bes. 91-92 u. 95-96; ders.: «Il dolore è il principio motore di tutto l'uman essere». Pietro Verri e Cesare Beccaria al di là della felicità, in: Piero Giordanetti, Giambattista Gori, Maddalena Mazzocut-Mis (a cura di): Il secolo dei Lumi e l'oscuro, Milano, Udine 2008, 71-84, hier 73-77; ders.: Lust, 


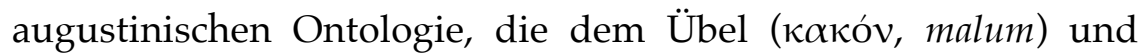
damit auch dem Leiden und dem Schmerz, der Krankheit und dem Tod jegliche ontologische Dignität abspricht, indem sie diese

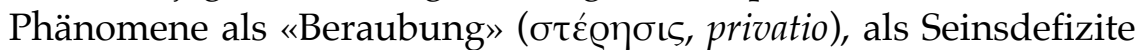
und letztlich als Nichtsein fasst ${ }^{34}$ - einer Ontologie, die das westliche philosophische Denken bis in die Neuzeit bestimmt hat und die erst von Hegel und Adorno überwunden wurde. ${ }^{35}$

Gottes Schöpfung ist für Verri also nicht, wie das jüdischchristliche Denken voraussetzt, gut und wie es die Litanei von Genesis 1 geltend macht, am Schluss sogar «sehr gut». ${ }^{36}$ Vielmehr sind für ihn das Übel, das Leiden und das Unbehagen (mal essere) jenes Sein, das die Grundbefindlichkeit des Menschen in der Welt kennzeichnet ${ }^{37}$ - jenes Unbehagen in der Kultur, das Freud diagnostiziert, weil das durch den jüdisch-christlichen Schöpfungsoptimismus aufgestellte «Programm des Lustprinzips, das den Lebenszweck setzt», nicht realisierbar ist, da «die Absicht, dass der Mensch 〈glücklich〉 sei, [...] im Plan der 〈Schöpfung〉 nicht enthalten ist». ${ }^{38}$

76-94, bes. 84-88; ders.: An den Grenzen hedonistischer Lebenskunst, 32-34; ders.: Facetten der Unlust, 23-28.

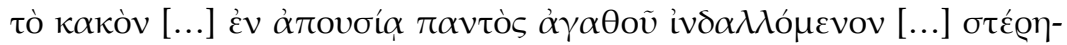
бıৎ Plot. enn. I 8 [51],1; «malum non esse nisi privationem boni usque ad quod omnino non est» Aug. conf. 3,12: «malumque [...] non est substantia, quia, si substantia esset, bonum esset» Aug. conf. 7,18.

Die einschlägigen Werke sind Hegels Wissenschaft der Logik (1812-1816), in der die Negativität Motor der Bewegung des Begriffs ist, auch wenn sie in der Bewegung immer aufhoben wird, und Adornos Negative Dialektik (1966), die eine Ontologie der Negativität ohne Hegels die christliche Heilsgeschichte reflektierende Versöhnungsdynamik begründet.

36 Der Schlussvers lautet: «Und Gott sah an alles, was er gemacht hatte, und siehe, es war sehr gut.» Gen 1,31.

37 P. Verri: Discorso sull'indole del piacere e del dolore, 107. Verri bezieht sich hier explizit auf Locke und zitiert den englischen Ausdruck «uneasiness»; vgl. John Locke: An Essay concerning Human Understanding, ed. P. H. Nidditch, Oxford 1975, II, XXI, 31-57.

Sigmund Freud: Das Unbehagen in der Kultur, Studienausgabe, IX 208. 
Auch wenn Verri nach seiner Analyse von Lust und Leiden der Menschen in der Welt die traurige Bilanz zieht, dass bei jedem Menschen die Summe erfahrenen Leidens grösser ist als die Summe der Lustempfindungen, dass ein Zustand eines dauerhaften Elends realistischer erscheint als die Utopie einer von Glück, Lust und Freude bestimmten Welt, ${ }^{39}$ ist seine Ontologie keineswegs pessimistisch oder gar lebensfeindlich, sondern sie begründet vielmehr den Willen zur Lust und den Willen zum Leben, der das theoretische Fundament seiner Anthropologie und Sozialphilosophie ist. Schmerzen und Leiden sind in ontologischer Perspektive «positive», das heisst nicht-defizitäre Negativität, sie sind «Motor» und «Antriebsprinzip» (principio motore) der menschlichen Entwicklung und Geschichte, ${ }^{40}$ also keine Privationen eines Guten, sondern vielmehr Bedingungen der Möglichkeit des Guten und damit von gleicher oder gar höherer ontologischer Dignität als das Gute.

In dieser Hinsicht kann Verri als Vorläufer der Existenzphilosophie gesehen werden. Leiden, Schmerz, Trauer und Krankheit sind keine Defizite, sondern bilden die Grundbedingung des menschlichen Daseins. Auf dieser Linie wird Kierkegaard das Scheitern der ästhetisch-hedonistischen Lebensform, also des auf Lust setzenden und zielenden Lebensentwurfs, als Voraussetzung für die Selbstwerdung und moralische Selbstwahl geltend machen: selbstbestimmtes Leben wird erst durch die Erfahrung des Scheiterns und Leidens möglich. Selbstentwurf und Selbstkonstitution des Menschen gelingen erst in und aus der Verzweiflung, zu der das Lustprinzip notwendig führt. Diese Verzweiflung, dieses Scheitern des hedonistischen Lebensentwurfs ist ein Schmerz und ein Leiden, die Kierkegaard mit den Wehen einer Gebärenden vergleicht. ${ }^{41}$ Kierkegaards Konzept der unter grossen Schmerzen vollbrachten Geburt des autonomen Selbst verabschiedet den zu

\footnotetext{
39 P. Verri: Discorso sull'indole del piacere e del dolore, $151 \mathrm{u} .101$.

40 «Il dolore precede ogni piacere, ed è il principio motore dell'uomo.» ebd., 131.

41 Vgl. Sören Kierkegaard: Entweder-Oder, übers. von Heinrich Fauteck, München 72003, 744-748 u. 762-771.
} 
Leere, Überdruss und Verzweiflung führenden Hedonismus - im Unterschied zu Verri, dessen Schmerz- und Leidensprimat das eudämonistische Aufklärungsdenken letztlich nicht dementiert, sondern in der utilitaristischen Formel vom grösstmöglichen Glück der grössten Zahl relativiert. ${ }^{42}$

Ich schliesse die Bemerkungen zur Ontologie von Lust und Leiden mit einigen Gedanken zum Tod. ${ }^{43}$ In der Theologie des 20. Jahrhunderts wurde im Anschluss an Heidegger ${ }^{44}$ die Frage diskutiert, ob der Tod bloss das Ende des (diesseitigen) Lebens sei oder vielmehr dessen Vollendung zum Ganzen. ${ }^{45}$ Dass diese Diskussion die ontologische Bedeutung des Todes möglicherweise nicht zu erfassen vermag, thematisiert Wolfhart Pannenberg in seiner Kritik an Heidegger und Karl Rahner. Pannenberg sieht im Tod gerade nicht die «Vollendung zum Ganzen», sondern vielmehr «den Abbruch des Daseins, seine Zerstörung»; die Möglichkeit der Vollendung hingegen besteht für ihn - «wenn überhaupt - nur jenseits des Todes». ${ }^{46}$ Das sich aus der theologisch-dogmatischen Perspektive ergebende Dilemma liesse sich allerdings vermeiden, wenn man die Endlichkeit des Lebens nicht als einen ontologi-

P. Verri: Discorso sulla felicità, Edizione Nazionale, III 241-242.

Vgl. dazu, allerdings mit teilweise anderen Akzentuierungen, W. Rother: An den Grenzen hedonistischer Lebenskunst, 38-39.

Martin Heidegger: Sein und Zeit, Tübingen ${ }^{131976, ~ 235-267 ~(« D a s ~ m o ̈ g-~}$ liche Ganzsein des Daseins und das Sein zum Tode»).

Für die erste Auffassung argumentiert beispielsweise Wolfhart Pannenberg: Tod und Auferstehung in der Sicht christlicher Dogmatik, in: ders.: Grundfragen systematischer Theologie, Gesammelte Aufsätze, Göttingen 1989, II 146-159 (vgl. hier 155: «Die These also, dass der Tod das Leben in seine Ganzheit bringe, ist zu bestreiten», 158: «jenseitige Vollendung»), für die letztere Karl Rahner: Zur Theologie des Todes, in: Zeitschrift für katholische Theologie 79/1 (1957) 1-44 (vgl. hier 16: «Und darum ist sein [des Menschen] Tod, das war das wichtigste Ergebnis unserer ersten Überlegung, die Einheit von Ende und Vollendung.» Vgl. auch die Position von Karl Jaspers, dem zufolge sich die Existenz zum Tode als «zu der notwendigen Grenze ihrer möglichen Vollendung» verhalte. K. Jaspers: Philosophie, II: Existenzerhellung, Berlin, Heidelberg 1956, 228.

W. Pannenberg: Tod und Auferstehung, 155. 
schen Mangel, sondern als integralen Bestandteil des Lebens und damit als Chance für ein gelingendes Leben betrachten würde. Dadurch wären wir genötigt, den Sinn des Lebens ausschliesslich im Diesseits zu suchen, was Pannenberg explizit ablehnt, ${ }^{47}$ doch lässt sich für eine solche diesseitige Perspektive auf der Grundlage biblischer Texte argumentieren, beispielsweise der Reflexionen des Apostels Paulus über die Kürze der Zeit und die Vergänglichkeit der Welt. ${ }^{48}$

Aus diesseitiger Perspektive gehört der Tod als Begrenzung des Lebens zu seiner Ganzheit. Insofern bedeuten Begrenzung und Grenze keinen Mangel, sondern sie definieren das Ganze, das sich durch die Grenze und als Begrenztes vom unendlichen Alles unterscheidet. Ohne den Tod wäre das Leben unbegrenzt und bliebe insofern immer unvollkommen und unvollendet. Der Tod ist daher nicht auf den Abbruch und den Verlust des Lebens zu reduzieren und auch nicht theologisch-metaphysisch als dessen Vollendung zu verklären, sondern als ein integral zum Leben gehörendes Ereignis zu fassen. Mit dieser ontologischen Integration jener Grenze in das Leben gelangt - statt des Endes - der endliche Prozess des Lebens in seiner Ganzheit in den Blick.

Dass die Endlichkeit des Lebens, seine durch Leiden und Krankheit gekennzeichnete Hinfälligkeit, keineswegs den Verlust der Möglichkeit eines gelingenden Lebens bedeutet, sondern im Gegenteil ein solches lustvolles Leben überhaupt erst ermöglicht, ergibt sich aus einem Gedanken Epikurs, der die Grenze zwischen Leben und Tod in aller Schärfe fasst. Für ihn sind Tod und Leben nicht vermittelt, sondern der Tod ist die absolute Negation des Lebens: Er ist das Nicht-Leben, das uns als Lebende nicht betrifft. ${ }^{49}$ In dieser Erkenntnis der Endlichkeit und Sterblichkeit unseres Lebens liegt die Bedingung der Möglichkeit eines gelingenden und

48

49

Ebd., 146.

1Kor 7, 29-31. Dazu W. Rother: Genießen und Genuss, 26-27.

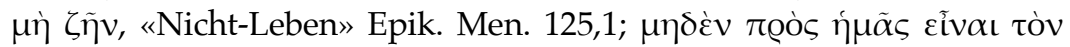
Өávatov, «dass der Tod uns nicht betrifft» Epik. Men. 124,3 (übers. von Jan-Erik Hessler). 
damit lustvollen Lebens. ${ }^{50}$ Würden wir ewig leben und wüssten wir dies, wären wir vor Krankheit und Leiden gefeit, könnten wir, da wir über unbegrenzte Zeit verfügen, alles aufschieben und würden uns so zu Tode langweilen, statt - wozu Seneca in seinen Reflexionen zur Zeit und zur Endlichkeit des Lebens, möglicherweise das «carpe diem» von Horaz aufgreifend, seinen Schüler Lucilius ermuntert ${ }^{51}$ - jede Stunde unseres Lebens auszukosten. ${ }^{52}$ Leiden, Krankheit und Tod, so sehr wir zweifellos gut daran tun, sie mit allen Mitteln zu bekämpfen, sind in dieser Perspektive keine Antagonisten der Lust, sondern ihre Möglichkeitsbedingungen.

conexus 1 (2018) 29-44

(C) 2018 Wolfgang Rother. Dieser Beitrag darf im Rahmen der Lizenz CC BY-NC-ND 4.0 - Creative Commons: Namensnennung/nicht kommerziell / keine Bearbeitungen - weiterverbreitet werden.

\section{https: / / doi.org/10.24445/ conexus.2018.01.005}

Prof. Dr. Wolfgang Rother, Universität Zürich, Philosophisches Seminar, Hirschengraben 56, 8001 Zürich wolfgang.rother@philos.uzh.ch

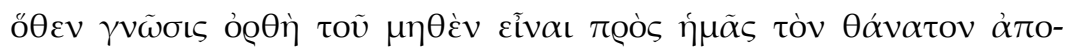

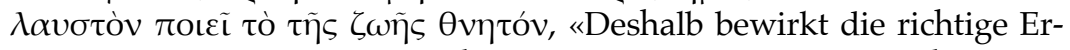
kenntnis, dass der Tod uns nicht betrifft, dass man die Sterblichkeit des Lebens geniessen kann» Epik. Men. 124,5 (übers. von J. E. Hessler). «carpe diem, quam minimum credula postero», «hasche den Tag, wenig Vertrau'n schenke dem morgenden!» Hor. carm. 1,11 (übers. Wilhelm Binder).

52 «Fac ergo, mi Lucili, quod facere te scribis, omnes horas complectere. Sic fiet ut minus ex crastino pendeas, si hodierno manus inieceris. Dum differtur, uita transcurrit.» «Tue also, mein Lucilius, was du zu tun schreibst - alle Stunden umfasse mit beiden Armen. So wirst du weniger vom Morgen anhängen, wenn du auf das Heute die Hand legst. Während es aufgeschoben wird, enteilt das Leben.» Sen. epist. 1,2 (übers. von Manfred Rosenbach). 